\title{
Las reclamaciones de los usuarios externos en un centro pediátrico de referencia nacional en Lima, Perú
} External user's complaints in a national reference pediatric center

\author{
Roberto Shimabuku ${ }^{1,2}$, Danitza Fernández ${ }^{1,2}$, Gladys Carlos ${ }^{1}$, Katia Granados ${ }^{1}$, \\ Ruth Maldonado ${ }^{1}$, Graciela Nakachi ${ }^{1,2}$ \\ Instituto Nacional de Salud del Niño (INSN), Lima, Perú. \\ ${ }^{2}$ Facultad de Medicina, Universidad Nacional Mayor de San Marcos (UNMSM), Lima, Perú.
}

\begin{abstract}
Resumen
Objetivo: Identificar las características de las reclamaciones de los usuarios externos de un centro pediátrico de referencia nacional. Diseño: Estudio descriptivo, retrospectivo. Institución: Instituto Nacional de Salud del Niño, Lima, Perú. Material: Libro de reclamaciones. Métodos: Del libro de reclamaciones del Instituto Nacional de Salud del Niño, entre julio 2011 y setiembre 2012, se elaboró una base de datos. El paciente podía tener más de un motivo de reclamación. Principales medidas de resultados: Datos demográficos, frecuencia, motivos, tiempo de resolución, personal aludido y resultados de las reclamaciones. Resultados: Se recolectó 283 copias de formatos que contenían 358 motivos de reclamaciones, siendo los más frecuentes: trato inapropiado $32,1 \%$, tiempo de espera prolongado 18,4\%, información deficiente 14,5\%, dificultad para el acceso en la atención 9,5\%, extravío de documentación 7,8\%, problemas de comunicación $7,5 \%$, privilegios $5,9 \%$, disconformidad con aspectos tangibles $4,2 \%$. Las áreas que recibieron más reclamaciones fueron: consultorios externos $41,7 \%$ (tasa de 0,5 por mil pacientes), vigilancia 9,2\%, Servicio Bajo Tarifario Diferenciado (privado) $9,2 \%$ y Caja 6,0\%. El $60 \%$ de las reclamaciones fueron resueltas y, de estas, el $84,2 \%$ antes de los 28 dias. En relación al personal de salud aludido en los reclamos estuvieron: médico $(26,4 \%)$, técnica de enfermería $(13,9 \%)$, personal administrativo $(12,2 \%)$, vigilante $(12,2 \%)$, personal de archivo $(9,7 \%)$, enfermera $(9,0 \%)$, cajero $(5,9 \%)$, admisionista $(2,1 \%)$. Conclusiones: Las caracteristicas de las reclamaciones sugieren intervenciones correctivas y educativas más apropiadas para así minimizar los reclamos de los usuarios 0 disminuirlas. Es necesario evaluar periódicamente las reclamaciones para aumentar la satisfacción de los usuarios.
\end{abstract}

Palabras clave: Reclamaciones, calidad de atención, satisfacción del usuario, hospitales especializados, niños, Lima, Perú.

\begin{abstract}
Objective: To identify complaints characteristics of external users in a national pediatric reference center. Design: Descriptive, retrospective study. Setting: Instituto Nacional de Salud del Niño (INSN), Lima, Peru. Material: Book of Complaints. Methods: A database was elaborated from the INSN Book of Complaints for the period July 2011 to September 2012. The patient could have had more than one complaint reason. Main outcome measures: Demographic data, frequency, motives, time of resolution, alluded personnel, and solution of complaints. Results: Two hundred and eighty-three formats were collected containing 358 complaints. Most frequent complaint reasons were: inappropriate attitude 32.1\%, long waiting time 18.4\%, deficient information $14.5 \%$, deficient access to services $9.5 \%$, loss of documentation (laboratory results or x-rays, formats, clinical records, among others) $7.8 \%$, problems in communication $7.5 \%$, privileged access to attention $5.9 \%$, objective aspects (facilities, appearance, cleaning, equipment) $4.2 \%$. The areas that received more complaints were: outpatient attention by physicians $41.7 \%$ (rate of 0.5 per thousand patients), security $9.2 \%$, private hospital service $9.2 \%$, and cashiers $6.0 \%$. Sixty per cent of claims were solved, and of these $84.2 \%$ were solved before 4 weeks since the complaint. Occupational groups generating complaints were: physician $(26,4 \%)$, nurse technician $(13,9 \%)$, administrative personnel $(12,2 \%)$, security personnel $(12,2 \%)$, archive personnel $(9,7 \%)$, nurse $(9,0 \%)$, cashier $(5,9 \%)$, admission $(2,1 \%)$. Conclusions: Complaints' characteristics suggest the need to take appropriate corrective and educational measures, so as to avoid or decrease them. Routine periodic evaluation of complaints is necessary to increase user's satisfaction.
\end{abstract}

Keywords: Complaints, quality of health care, user's satisfaction, hospitals, specialized hospitals, child, Lima, Peru.

An Fac med. 2014;75(3):245-50 / doi: http://dx.doi.org/10.15381/anales.v75i3.9779

\section{INTRODUCCIÓN}

En general, en los hospitales especializados del Perú se realizan periódicamente encuestas para evaluar la satisfacción del usuario externo ${ }^{(1,2,3)}$, aunque no tenemos reportes, locales o nacionales, sobre reclamaciones escritas por parte de los usuarios sobre el servicio recibido a nivel hospitalario.
En el año 2011 se aprobó la norma legal D.S. 042-2011-PCM ${ }^{(4)}$, en el cual se establece la atención de reclamos mediante el Libro de Reclamaciones, el que se operativiza con una Directiva Interna en el Instituto Nacional de Salud del Niño.

La calidad en los servicios de salud y satisfacción del paciente están estre- chamente relacionados ${ }^{(5,6)}$. Por otra parte, la mala calidad de la atención se asocia con insatisfacción del paciente, lo que puede expresarse a través de reclamaciones. Pero hay pacientes que no reportan la reclamación y simplemente no vuelven, y su insatisfacción puede ser trasmitida a amigos o parientes, con pérdida del prestigio institucional o de potenciales nuevos pacientes ${ }^{(7)}$. 
Como ha sido señalado por Allen y col ${ }^{(8)}$, las encuestas no revelan la fuente o causa del problema, solo que existen. Pero las reclamaciones por escrito de los usuarios pueden expresar directamente por qué y cómo son afectados en el establecimiento de salud, y estimamos que representan una fuente de información útil que debe ser analizada. Este estudio de las reclamaciones junto con las encuestas de satisfacción ofrecerá un cuadro más preciso de las insatisfacciones de los usuarios.

El objetivo del estudio fue identificar las características de las reclamaciones escritas de los usuarios externos de un centro pediátrico de referencia nacional.

\section{MÉTODOS}

Se diseñó un estudio descriptivo, retrospectivo, revisando el Libro de Reclamaciones del INSN, el cual se basó en el Procedimiento para que el Usuario Externo inicie un Reclamo en la Institución.

Tratándose de niños de menores de edad, todas las reclamaciones fueron hechas por los padres, familiares o apoderados de los pacientes o usuario externo, que podían tener más de un motivo de reclamación.

El Padre o Tutor del paciente presentan el Reclamo en el Formato del Libro de Reclamaciones, el cual es tipificado previo análisis y se remite al responsable del Área donde se originó el Reclamo, teniendo un plazo de 20 días para dar una acción correctiva.

Los jefes de Áreas dan a conocer por escrito las acciones correctivas de acuerdo al análisis del Reclamo. En los textos de los reclamos se encuentran expresiones variadas tales como "falta de sencillo para dar vuelto", "no disponibilidad de la Historia Clínica", "exámenes auxiliares o informes radiográficos no oportunos en la historia clínica", "falta de medicamentos en el área de Farmacia de Emergencia", "mejora en la limpieza de los servicios higiénicos", "desabastecimiento de jabón líquido en los baños", "operatividad del surtidor de pañales" y "devoluciones de dinero cuando se ha reportado error de digitación", entre otros

La respuesta al Reclamo se hace a través del teléfono o correo electrónico registrado en la copia del Reclamo, en un plazo no mayor de 30 días de presentado este.

De la información suscrita por los responsables del paciente en el Libro de Reclamaciones se elaboró una base de datos, que abarca desde julio de 2011 a setiembre de 2012.

Se cuenta con un solo Libro de Reclamaciones en todo el INSN; prospectivamente contiene las reclamaciones escritas que en forma espontánea los usuarios realizan a mano.

Alrededor de 10\% de las reclamaciones tiene dificultad en su lectura por deficiente ortografía y caligrafía.

El Reglamento que aprueba el Libro de Reclamaciones fue establecido mediante Decreto Supremo № 042-2011PCM, publicado en el diario El Peruano, el 8 de mayo del $2011^{(4)}$, y se implementó desde el mes de julio de 2011. La misma norma legal incluye el modelo de formato válido a nivel nacional. Hasta entonces existían las quejas verbales, o escritas a través de los buzones de sugerencias, con un formato distinto al actual, o más raramente por teléfono o correo electrónico.

Las reclamaciones fueron atendidas por la Oficina de Gestión de la Calidad, según procedimiento establecido, recibiendo las medidas correctivas realizadas por los jefes de Área donde se originó el reclamo, descargos o réplicas del personal aludido e informando lo actuado a los usuarios por teléfono o correo electrónico.

Los reclamos de cada usuario fueron introducidos en una base de datos junto con los motivos de reclamaciones. Los datos registrados fueron: nombre, parentesco, procedencia, motivo de la reclamación, área donde ocurrió, personal aludido, fecha de la reclamación y fecha de la respuesta del área responsable.

En relación al motivo de las reclamaciones, establecimos las siguientes: trato inapropiado, tiempo de espera prolongada, información deficiente, dificultad para el acceso en la atención, extravío de documentación (resultados de laboratorio o rayos X, formularios, historias clínicas, entre otros), problemas de comunicación, privilegios, disconformidad con aspectos tangibles (facilidades, apariencia, limpieza, equipos). Las reclamaciones fueron discutidas caso por caso por los autores, llegándose a un consenso final a través de la narrativa escrita del usuario, con el fin de estandarizar el motivo de reclamo. No se ha encontrado una taxonomía o codificación estándar sobre los motivos de las reclamaciones en nuestro medio.

\section{Glosario:}

- Reclamación: disconformidad relacionada a los productos o servicios ${ }^{(9)}$.

- Encuesta: es una herramienta para evaluar el nivel de satisfacción del usuario externo sobre la calidad de servicio que se le brinda ${ }^{(6)}$.

- Tiempo de espera: promedio de minutos que transcurren entre el momento en que el usuario solicita la atención en el servicio y el inicio de la consulta médica de esta por el médico. El tiempo de espera es prolongado cuando supera el estándar de 45 minutos en consulta externa, y en emergencia según estándar establecido para servicios de emergencia ${ }^{(10,11)}$.

- Privilegios: mide la percepción si existe trato discriminatorio o preferencial por algunos usuarios. Será categorizado como privilegio cuando el usuario refiere que ingresan usuarios o familiares de nuestro personal interrumpiendo el orden programado.

- Dificultad para el acceso en la atención: incapacidad para cumplir con el servicio ofrecido o insuficiente oferta para la demanda. 


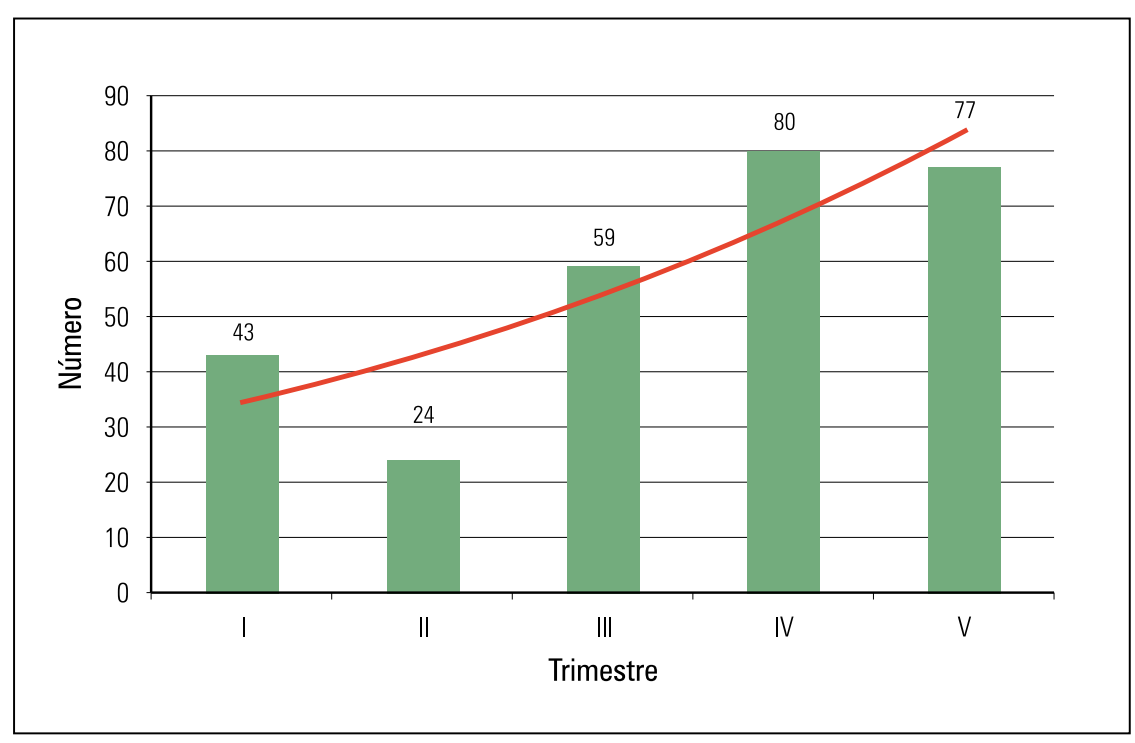

Figura 1. Frecuencia trimestral de las reclamaciones.

- Problemas en la comunicación: dificultad del personal para comunicarse con el usuario y ocurre cuando el usuario refiere que no le contestan las preguntas o no entiende lo que le explican por la atención recibida.

- Extravío de la documentación: demora en la ubicación de historias clínicas o resultados de los exámenes de laboratorio, rayos $\mathrm{X}$ y formatos administrativos indispensables para la atención del paciente.

- Disconformidad con aspectos tangibles: percepción negativa de infraestructura física, equipamiento, limpieza o apariencia del personal.

\section{RESULTADOS}

Los acompañantes de los usuarios en $71 \%$ fueron madres y $93 \%$ de los usuarios fueron de Lima. La frecuencia trimestral de reclamaciones fue: 43, 24 , 59,80 y 77 del primero al quinto trimestre, respectivamente (figura 1).

Se recolectó 283 formatos que contenían 358 reclamaciones, siendo los motivos más frecuentes: trato inapropiado $32,1 \%$, tiempo de espera prolongada 18,4\%, información deficiente $14,5 \%$, dificultad para el acceso en la atención 9,5\%, extravío de documentación (resultados de laboratorio o rayos $\mathrm{X}$, formularios, historias clínicas, entre otros) $7,8 \%$, problemas de comunicación 7,5\%, privilegios 5,9\%, disconformidad con aspectos tangibles (facilidades, apariencia, limpieza, equipos) $4,2 \%$ (tabla 1). Las áreas que recibieron más reclamaciones fueron: consultorios externos $41,3 \%$ (tasa de 0,5 por mil pacientes), vigilancia 9,1\%, SBTD (Servicio Bajo Tarifario Diferenciado, con tarifas similares a clínicas privadas) 9,1\% y Caja (lugar donde se efectúa el pago de servicios) 5,9\% (tabla 2).

En relación al personal de salud aludido en los reclamos, corresponde en

Tabla 1. Motivos de reclamaciones $(n=358)$.

\begin{tabular}{lcc} 
Motivos & $\mathrm{n}^{\circ}$ & $\%$ \\
\hline Trato inapropiado & 115 & 32,1 \\
Tiempo de espera prolongado & 66 & 18,4 \\
Información deficiente & 52 & 14,5 \\
$\begin{array}{l}\text { Dificultad para el acceso en la } \\
\text { atención }\end{array}$ & 34 & 9,5 \\
Extravío de documentación & 28 & 7,8 \\
Problema de comunicación & 27 & 7,5 \\
Privilegios & 21 & 5,9 \\
Disconformidad con aspectos & 15 & 4,2 \\
tangibles & & \\
\hline
\end{tabular}

Tabla 2. Áreas con mayor frecuencia de reclamaciones $(n=286)$.

\begin{tabular}{lcc} 
Áreas & $\mathrm{n}^{\circ}$ & $\%$ \\
Consultorios externos & 118 & 41,3 \\
Vigilancia & 26 & 9,1 \\
Servicio bajo tarifario diferenciado & 26 & 9,1 \\
Caja & 17 & 5,9 \\
Archivo & 16 & 5,6 \\
Apoyo al diagnóstico & 16 & 5,6 \\
Emergencia & 11 & 3,8 \\
Hospitalización & 10 & 3,5 \\
Admisión & 10 & 3,5 \\
Unidad de Seguro Integral de Salud & 8 & 2,8 \\
Otros & 28 & 9,8 \\
\hline
\end{tabular}

$38,1 \%$ a personal profesional de la salud, seguido de aquel personal que ofrece el primer contacto con la institución y que representan el 21,5\%: vigilante, cajero, admisionista y orientadora; en algunos casos el usuario no especificó al personal de salud, por lo que se registró como personal de laboratorio y de diagnóstico por imágenes (tabla 3).

Tabla 3. Personal de salud aludido en las reclamaciones.

\begin{tabular}{lcc} 
Personal de Salud & $\mathrm{n}^{\circ}$ & $\%$ \\
Médico & 76 & 26,4 \\
Técnica de enfermería & 40 & 13,9 \\
Personal administrativo & 35 & 12,2 \\
Vigilante & 35 & 12,2 \\
Personal de archivo & 28 & 9,7 \\
Enfermera & 26 & 9,0 \\
Cajero & 17 & 5,9 \\
Admisionista & 6 & 2,1 \\
Tecnólogo médico & 4 & 1,4 \\
Digitador & 4 & 1,4 \\
Orientadora & 4 & 1,4 \\
Personal de Laboratorio & 3 & 1,0 \\
Cafetería & 3 & 1,0 \\
Personal de Dx. imágenes & 3 & 1,0 \\
Odontólogo & 2 & 0,7 \\
Químico Farmacéutico & 1 & 0,3 \\
Trabajadora social & 1 & 0,3 \\
\hline
\end{tabular}




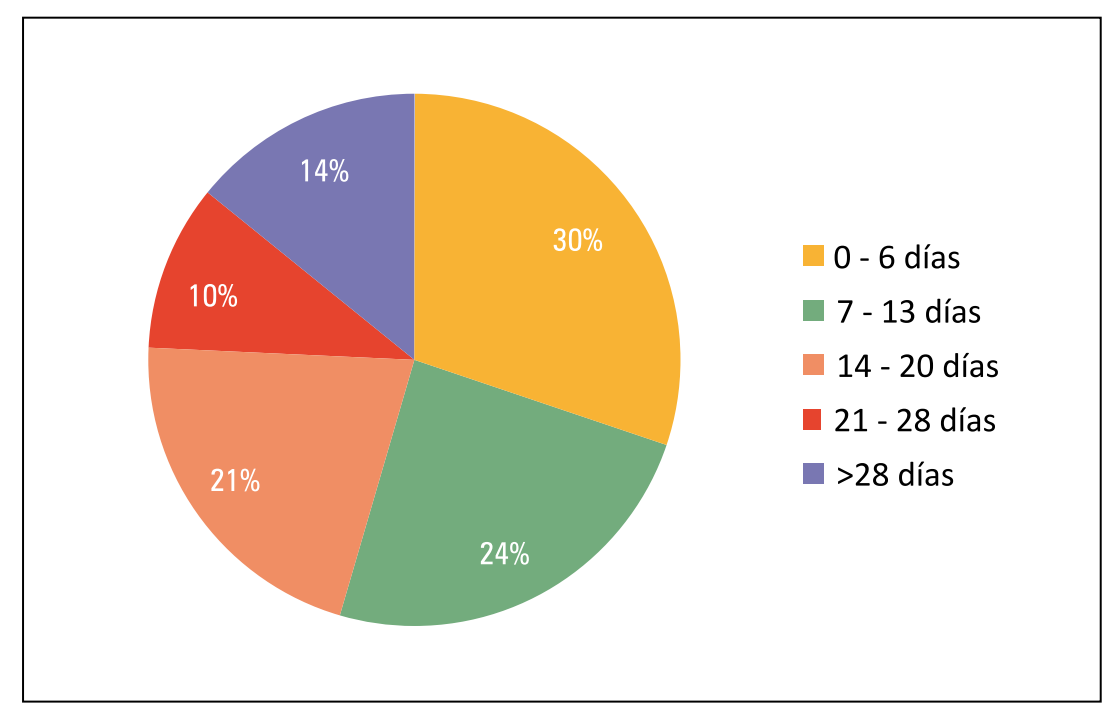

Figura 2. Tiempo de resolución de las reclamaciones.

La figura 2 muestra el tiempo de resolución de las reclamaciones medidas en días. El $60 \%$ de las reclamaciones fueron resueltas, y de estas el 84,2 \% antes de los 28 días, aunque la norma establece como plazo máximo 30 días.

Nuestra tasa de reclamaciones fue 0,5 por cada 1000 atenciones, en la atención ambulatoria.

De la revisión de los casos, luego de resueltos, hemos encontrado que aproximadamente $80 \%$ de las Reclamaciones estaban justificadas, siendo las no justificadas aquellas en las que el personal aludido presentó un descargo razonable y a su debido tiempo, quedando estas últimas observadas y como antecedentes.

\section{DISCUSIÓN}

El Instituto Nacional de Salud del Niño de Lima es el único establecimiento del país dedicado exclusivamente a la atención de niños y adolescentes hasta los 18 años de edad. Tiene 438 camas disponibles, más de 40 subespecialidades pediátricas, 411 médicos, 610 enfermeras y 588 técnicas asistenciales. Se atiende las 24 horas del día, en todas las subespecialidades, incluyendo guardias y retenes. Hay aproximadamente
45000 atenciones ambulatorias al mes. En el año 2012, se registró 314664 atenciones médicas en Consultorio Externo, 10368 egresos en el Área de Hospitalización, 55902 atenciones en el Área de Emergencia y se registró en Cirugía Mayor 5917 intervenciones, y en Cirugía de Día 5014 intervenciones quirúrgicas ${ }^{(12)}$.

En la literatura médica se encuentra que son diversas las personas que formulan las reclamaciones, ya sea por los mismos pacientes, parientes, apoderados o amigos o, como en nuestro caso, las reclamaciones son presentadas por los padres.

También se usa diversas modalidades de exponer una reclamación: reclamaciones verbales, reclamaciones escritas, cartas, teléfono, fax, correo electrónico, encuestas de satisfacción del usuario, reclamaciones solicitadas al usuario, entrevistas cualitativas, visitas a pacientes por el equipo de mejora de la calidad, libro de reclamaciones, o una combinación de ellas ${ }^{(13)}$.

En nuestro establecimiento, las reclamaciones se recibían a través del buzón de sugerencias y formato de quejas institucional, las que eran atendidas y resueltas por personal de la Oficina de Gestión de la Calidad.
Actualmente se usa el Libro de Reclamaciones con formatos que permiten comentarios escritos, aunque aún subsisten las reclamaciones verbales. En nuestro libro hay reclamaciones en la que no hemos podido precisar el parentesco con el paciente, lo cual no es infrecuente ${ }^{(14)}$.

Llama la atención que nuestro servicio de Emergencia tenga menos reclamaciones que otros sectores del establecimiento. Una explicación podría ser que el Libro de Reclamaciones esté situado en el sector de consulta externa, mientras que la Emergencia se encuentra aproximadamente a 200 metros de distancia. Sin embargo, el servicio bajo tarifario diferenciado, conocido como 'la clínica' que se encuentra a similar distancia tiene más del doble de reclamaciones. En este caso, los usuarios de 'la clínica' tendrían mayor sustento para sus reclamaciones por el mayor gasto de bolsillo. Probablemente pertenecen a un mejor estrato socioeconómico, con mejor nivel educativo y mejor conocimiento de sus derechos.

El trabajo de Lim y col ${ }^{(15)}$, en Singapur, con reclamaciones verbales (64\%) y escritas $(36 \%)$ tiene una tasa bastante baja $(0,04$ reclamaciones por 1000 atenciones), y basada en atención primaria, de capa simple, revela que los motivos de sus reclamaciones son similares a otros reportes: trato inapropiado, falta de habilidades profesionales, no responder a las expectativas, tiempo de espera y comunicación. Uno de los estudios más grandes ${ }^{(16)}$ describe una tasa de reclamaciones $(0,4$ por 1000 pacientes) semejante al nuestro en consulta ambulatoria, y en general todas las áreas hospitalarias el 85\% por ciento de las reclamaciones fueron resueltas fácilmente. El trabajo de Kline y col ${ }^{(17)}$ revisa el tema de predictibilidad de las reclamaciones y lo asociaron con los casos de más alta complejidad clínica, sometidos a muchos procedimientos o con largas estancias.

Nuestro estudio recoge las reclamaciones por escrito de los pacientes, o mejor dicho de los padres, familiares 
o apoderados de los niños y adolescentes. No sabemos cuánto de las reclamaciones no son reportadas, y usan otros medios para realizarlas, como las comunicadas a la Defensoría de Salud, Voluntariado, Orientadoras, Jefaturas, sección Informes, entre otras.

No tenemos reportes nacionales para comparar nuestros resultados. Conocer los motivos de las reclamaciones es el primer paso necesario para prevenirlas y manejarlas. Establecer categorías o motivos de las reclamaciones no es una tarea sencilla. El trabajo de Montini y col ${ }^{(13)}$ busca establecer una taxonomía estándar para las reclamaciones de los pacientes, incluyendo hacia qué grupo ocupacional va dirigido. Por otro lado, Allen y col ${ }^{(8)}$ han reunido las reclamaciones (comentarios negativos) de las encuestas y realizan un seguimiento de las mismas para resolverlas.

El primer motivo de reclamaciones que hemos encontrado es el trato inapropiado. Los pacientes esperan ser tratados por el personal, en especial el médico, con cortesía, empatía y compasión, no con rudeza o descuido. Por el contrario, puede ocurrir que el paciente malinterprete la conducta del personal como hostil. El trabajo de Beckman ${ }^{(18)}$, tomando como base de datos casos de médicos que fueron llevados a juicios, identifica hasta 4 categorías de reclamaciones: entrega disfuncional de información, falta de comprensión de la perspectiva de pacientes y/o familiares, desacreditación (desvalorización) de los puntos de vista de paciente $y / o$ familiares, y deserción (comunicación ausente).

El trabajo de Moore y col (19) describe cómo desde el pregrado se puede incluir cursos formales en habilidades comunicacionales, en algunos programas de posgrado y adicionalmente cursos para los docentes clínicos sobre cómo enseñar la comunicación médico paciente.

El segundo motivo es el tiempo de espera prolongado, una reclamación que hemos encontrado en una encuesta de nuestro establecimiento ${ }^{(1)}$, que lo reportan largo o muy largo el 65,6\% de los encuestados en la consulta externa. En contraste con el servicio de emergencia, donde solo $1,6 \%$ de los pacientes encontró el tiempo de espera muy largo, y largo el $19,4 \%$. Pero el tiempo de espera en Emergencia es un tema prioritario debido a que la expectativa es que la atención sea inmediata (20), más evidente con entrevistas cualitativas, en profundidad, cara a cara; los usuarios opinan que ellos requieren más explicaciones sobre la priorización de la atención ${ }^{(21)}$.

El trabajo de Montini y col ${ }^{(13)}$, efectuado en dos grandes hospitales de Boston, pero basado en 8 estudios previos, revela contra qué personas eran las reclamaciones: médicos o dentistas 22\%, enfermeras $12 \%$, personal de apoyo o administrativo $11 \%$, tecnólogos médi$\cos 8 \%$, personal en general o no especificado $47 \%$. No menciona a las técnicas de enfermería. Que el médico sea el personal más aludido no es casual, ya que es considerado el responsable último del proceso asistencial, incluyendo aspectos administrativos o de circuitos internos.

La relación médico-paciente juega un rol importante en el cuidado del paciente y una buena comunicación está relacionada a la satisfacción del paciente y también de los médicos. Hay decisiones más acertadas y mejor adherencia al tratamiento ${ }^{(22)}$.

El trabajo de Tam y col ${ }^{(23)}$ respalda que la comunicación insuficiente es la causa mayor de insatisfacción del paciente en el departamento de Emergencia en Hong Kong, al revisar 3 años de reclamaciones. Revela que solo $59 \%$ de las reclamaciones fueron juzgadas como válidas en un departamento de emergencia. Este estudio enfatiza la necesidad de mejorar las habilidades comunicacionales y aumentar la supervisión y entrenamiento con personal senior las 24 horas del día. El estudio de Taylor ${ }^{(16)}$ señala también que fallas en la comunicación del personal es una causa común en las reclamaciones.

Por otro lado, también existe la reclamación encubierta ${ }^{(24)}$, que no es comunicada por escrito en el Libro de Reclamaciones y es resuelta por la defensoría de la salud, del voluntariado, orientadoras y por personal del INSN, tanto asistencial como administrativo, casi siempre en forma anónima y discreta. No sabemos cuánto representan del total, pero se ha estimado que solo entre 17 y $20 \%$ de las reclamaciones son presentadas formalmente ${ }^{(24)}$. Allen y col ${ }^{(8)}$ han encontrado que solo $25 \%$ de los pacientes hospitalizados llenan encuestas y escriben comentarios adicionales con reclamaciones o sugerencias de mejora.

La entidad pública está obligada a dar respuesta al usuario, por medios físicos o electrónicos, en un plazo máximo de 30 días hábiles, informándole, de ser el caso, acerca de las medidas adoptadas para evitar el acontecimiento de hechos similares en el futuro ${ }^{(4)}$.

Hay algunas limitaciones de nuestro estudio. A alrededor de $10 \%$ de las hojas de reclamaciones le faltaban datos o eran ilegibles, por lo que es posible algún sesgo en la elaboración de la base de datos. No tenemos una definición precisa de los motivos. Los motivos de las reclamaciones han sido clasificados revisando caso por caso por los autores, por lo que puede haber errores en su clasificación.

Una recomendación es que en estudios similares debe mejorarse la definición de los motivos de reclamación y así estandarizarlos y ser comparables.

La conclusión del estudio es que las características de las reclamaciones observadas sugieren intervenciones correctivas y educativas más apropiadas para evitarlas o disminuirlas. Es necesario continuar evaluando periódicamente las reclamaciones, seguir resolviéndolas y así mejorar la calidad del servicio.

\section{RECONOCIMIENTOS}

Los autores agradecen al Dr. Carlos Álamo por su apoyo en la búsqueda bibliográfica. Todos los autores participa- 
ron en la concepción del estudio. R.M., K.G., G.C., D.F. en la elaboración de la base de datos, y R.S, D.F., R.M. en la discusión de los datos, y todos en la redacción del manuscrito final. Este estudio fue autofinanciado.

\section{REFERENCIAS BIBLIOGRÁFICAS}

1. Shimabuku R, Huicho L, Fernández D, Nakachi G Maldonado R, Barrientos A. Niveles de insatisfacción del usuario externo en el Instituto Nacional de Salud del Niño de Lima, Perú. Rev peru med exp salud publica. 2012; 29(4):483-9.

2. Seclén-Palacin J, Darras C. Satisfacción de usuarios de los servicios de salud: factores sociodemográficos y de accesibilidad asociados. Perú, 2000 An Fac med. 2005; 66(2):127-41.

3. MINSA. Perú: Proyecto 2000. Satisfacción del usuario. Estudio en tres Hospitales de Trujillo y Arequipa. Lima, 2002.

4. Presidencia del Consejo de Ministros, DS No. 0422011-PCM, 8 de mayo del 2011.

5. MINSA. Perú. Documento técnico "Sistema de Gestión de la Calidad en Salud. RM N 519-2006/ MINSA.

6. MINSA. Perú. Guía Técnica para la Evaluación de la Satisfacción del usuario Externo en los Establecimientos de Salud y Servicios Médicos de Apoyo. RM N ${ }^{\circ}$ 527-2011/MINSA. Publicada en el Diario Oficial "El Peruano" el 14 de julio de 2011.

7. Kessler S. Measuring and managing customer satisfaction: going for the gold. Milwaukee: ASQC Quality Press, 1996.

8. Allen LW, Creer E, Leggitt M. Developing a patient complaint tracking system to improve performance . Jt Comm J Qual Improv. 2000; 26(4):217-26.

9. Presidencia del Consejo de Ministros, DS No. 011 2011-PCM, 22 de abril del 2011

10. MEF. Directiva General del Sistema Nacional de Inversión Pública. Resolución Directoral $N^{\circ} 003-$ 2011-EF/68.01. Publicada en el Diario Oficial "E Peruano" el 09 de abril de 2011.

11. MINSA. Norma Técnica de los Servicios de Emergencia. NTS 042/MINSA/DGSP/V.01. RM No386-2006/MINSA. Publicada en el Diario Oficial "El Peruano" el 20 de abril de 2006.

12. Instituto Nacional de Salud del Niño. Análisis de la Situación de Salud (ASIS) del INSN, 2013, Lima, Perú.

13. Montini T, Noble AA, Stelfox HT. Content analysis of patient complaints. Int J Qual Health Care. 2008; 20(6):412-20.

14. Domingo A, Luaces C, Mañosa A, García JJ, Serra M, Pou J. Evaluación de las reclamaciones presentadas en un servicio de urgencias pediátricas en un hospital materno-infantil. Rev Calidad Asistencial. $2001 ; 16: 404-8$

15. Lim HC, Tan CB, Goh LG, Ling SL. Why do patients complain ? A primary health care study. Singapore Med J. 1998; 39(9):390-5.

16. Taylor DM, Wolfe RS, Cameron PA. Analysis of complaints lodged by patients attending Victorian hospitals, 1997-2001. Medical J Aust. 2004; 181(1):31-5.

17. Kline TJB, Willness C, Ghali WA. Predicting patient complaints in hospital settings. Qual Saf Health Care. 2008; 17:346-50. doi:10.1136/ qshc.2007.024281.

18. Beckman HB, Markakis KM, Suchman AL, Frankel RM. The doctor-patient relationship and malpractice. Lessons from plaintiff depositions. Arch Intern Med.1994; 154(12):1365-70.
19. Moore P, Vargas A, Nuñez S, Macchiavello S Un estudio de reclamos hospitalarios: el rol de la relación médico-paciente. Rev méd Chile. 2011; 139(7):880-5.

20. Wong LL, Ooi SBS, Goh LG. Patients'complaints in a hospital emergency department in Singapore. Singapore Med J. 2007; 48(11):990-5.

21. Cross E, Goodacre S, O'Cathain A, Arnold J. Rationing in the emergency department: the good, the bad and the unacceptable. Emerg Med J. $2005 ; 22: 171-8$.

22. Silverman J, Kurtz SM, Draper J. Skills for Communicating with Patients. Second ed. Abingdon: Radcliffe Medical Press; 2005.

23. Tam AYB, Lau FL. A three-year review of complaints in emergency department. Hong Kong j emerg med. 2000; 7:16-21.

24. Corbacho MS, Garcia-Mata JR, Blecua MJ. La reclamación encubierta. Identificando problemas de calidad. Rev Calidad asistencial. 2001; 16:29-34.

Artículo recibido el 27 de marzo de 2014 y aceptado para publicación el 30 de junio de 2014.

\section{Correspondencia:}

Dr. Roberto Shimabuku

Instituto Nacional de Salud del Niño

Correo electrónico: rshimabuku@insn.gob.pe

Teléfono: 3300033 Celular: 999269938

Nota: un reporte preliminar fue presentado en las XII Jornadas Cientificas Sanfernandinas, organizado por la Facultad de Medicina, del 13 al 17 de agosto del 2013; Lima, Perú. 